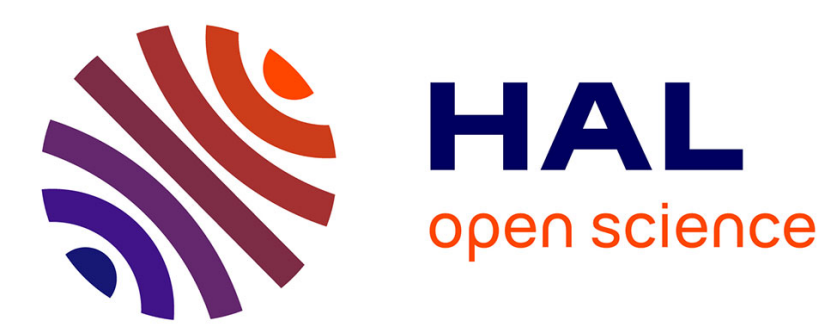

\title{
Compressive Template Matching on Multispectral Data
} Sylvain Rousseau, David Helbert, Philippe Carré, Jacques Blanc-Talon

\section{To cite this version:}

Sylvain Rousseau, David Helbert, Philippe Carré, Jacques Blanc-Talon. Compressive Template Matching on Multispectral Data. International Conference on Acoustics, Speech, and Signal Processing, May 2013, Vancouver, Canada. pp.ID2697. hal-00798439

\section{HAL Id: hal-00798439 \\ https://hal.science/hal-00798439}

Submitted on 16 Jan 2014

HAL is a multi-disciplinary open access archive for the deposit and dissemination of scientific research documents, whether they are published or not. The documents may come from teaching and research institutions in France or abroad, or from public or private research centers.
L'archive ouverte pluridisciplinaire HAL, est destinée au dépôt et à la diffusion de documents scientifiques de niveau recherche, publiés ou non, émanant des établissements d'enseignement et de recherche français ou étrangers, des laboratoires publics ou privés. 


\title{
Compressive Template Matching on Multispectral Data
}

\author{
Sylvain Rousseau*, David Helbert*, Philippe Carré*, Jacques Blanc-Talon ${ }^{\dagger}$ \\ *XLIM Laboratory, UMR CNRS 7252, University of Poitiers, France \\ ${ }^{\dagger}$ DGA/D4S/MRIS Bagneux, France
}

\begin{abstract}
This paper adapts a new template matching and target detection algorithm in multispectral images to a compressive sensing strategy. That template matching algorithm found in [1] relies on particular properties of $L_{1}$ minimization algorithms to succeed. We propose a new algorithm that is reconstructing in a single step the location of a given signature of interest bypassing the image reconstruction and the template matching algorithm on that image. For that purpose, we use a modified split Bregman algorithm with various regularizers. We conduct numerical experiments on real-world multispectral image.
\end{abstract}

Index Terms-multispectral image, compressed sensing, template matching, Bregman

\section{INTRODUCTION}

$\mathbf{T}$ ARGET detection is one of the most important applications of multispectral imaging. Processing the data can become very time consuming and complex. There are many target detection techniques. Recently Guo and Osher proposed an original approach relying on $L_{1}$ minimization in [1]. The data collected by multispectral sensors is modeled as a matrix $X$. Each column corresponds to a channel and each row is a pixel's spectrum. Suppose we want to detect pixels of spectral signature $s$. The following minimization in [1] is suggested:

$$
\underset{u \geq 0}{\arg \min }\|u\|_{1} \quad \text { s.t. } \quad\left\|X^{T} u-s\right\|_{2}<\sigma .
$$

The pixels of spectral signature $s$ in $X$ are identified by the nonzero entries in $u$. This can be intuitively explained as follows. The term $X^{T} u$ is the linear combination of all pixels' spectrum of $X$ weighted by $u$. The regularizer used is the $L_{1}$ norm that make $u$ sparse. As a result, the algorithm is looking for a reduced set of pixels whose linear combination with coefficients in $u$ yields $s$. The only way to satisfy this constraint is to have a nonzero entry in $u$ at every pixels of spectral signature $s$. That way, we would have a linear combination of spectral signature approaching $s$ which would give $s$.

However it raises two questions:

- How can we be sure that all pixels of interest are spotted? This raises the question of missing detection.

- A linear combination of random pixels could yield $s$. How can we be sure such a combination is discarded? This raises the question of false positive.

In the following, we will give arguments that answer these two questions.

The outline of the paper is as follows. In section II we review the template matching algorithm and answer questions raised in the introduction. In section III we develop a compressive template matching performing the same detection without having the multispectral image at hand. Section IV is devoted to numerical results applied to two different multispectral images. We give conclusions and perpectives in section V.

\section{Template Matching}

The first question is why is there no false negative? In other words, why every pixel of interest has a nonzero corresponding entry in $u$ ? This can be intuitively explained by noting that if a solution of $X^{T} u=s$ minimizing the $L_{1}$ norm is randomly selected then it has with high probability the largest possible support. Indeed, the $L_{1}$-ball is a polytope: it is a bounded intersection of half-spaces. Its boundary is composed of $d$ dimensional faces. Edges are 0 -dimensional faces and faces are just $(n-1)$-dimensional faces. The $L_{1}$-ball has the property that a point on its boundary and of support $d$ is on a $(d-1)$ face. Now, the solutions of $X^{T} u=s$ form a affine subspace. Those whose $L_{1}$ norm is minimum belongs to the intersection of this subspace with the boundary of a $L_{1}$-ball of minimum radius. A point chosen at random in that intersection belongs to a face with high probability. Thus, it has the highest support. Based on this remark we make the following assumption:

Assumption II.1. Algorithms used to solve minimization problem like (1) converge to a solution that has the largest $L_{0}$ norm among those that have the smallest $L_{1}$ norm.

We now formulate a proposition that is easily derived from the previous assumption and shows that two different solutions with maximal support actually have the same support.

Proposition II.1. Let $u_{1}$ and $u_{2}$ be two solutions of the minimization problem (1) whose $L_{0}$ norm is the largest. Then their support are equal.

This proves that the algorithms will find the same support and that this support will be maximal, hence no false negative.

The second question is why is there no false positive? In other words why $u$ has to be nonzero only for pixels of signature $s$ ? Indeed in practice $X$ has many more rows than columns so $X^{T}$ is likely surjective. In that case, we can imagine that $u$ could mix very different signatures so that $X^{T} u=s$. However, even if such a combination exists, the corresponding $L_{1}$ norm of $u$ would be likely larger than 1 and thus discarded. 


\section{Compressive Template Matching}

The previous template matching algorithm needs the whole multispectral data cube to work. In this section, we propose an new algorithm working in a compressive sensing context [2], [3] where we only have access to a small number of linear measurements on the multispectral image. The idea is to directly reconstruct the vector $u$ solution of (1) rather than reconstructing the whole multispectral image first and then solve the algorithm (1). A similar bypassing idea has been exploited on a recent paper [4] which deals with unmixing of hyperspectral data.

We introduce necessary notations here. We will assume that the multispectral image has $n_{P}$ pixels and $n_{B}$ bands. As a result, the matrix $X$ has $n_{P}$ rows and $n_{B}$ columns. The acquisition model is described as

$$
M=F X,
$$

where $F$ is a sensing matrix of size $m \times n_{P}$ and $M$ the measurement matrix of size $m \times n_{B}$. Suppose we only acquire a fraction $p$ of the overall data. We have the relation

$$
m \cdot n_{B}=p \cdot n_{B} \cdot n_{P} .
$$

so the number of rows of $F$ is $m=\left\lfloor p \cdot n_{P}\right\rfloor$.

We now have to eliminate $X$ from the two equations $X^{T} u=s$ and $M=F X$. One way is to introduce a matrix between $X^{T}$ and $u$ of the from $F^{T} A$ so we could replace $X^{T} F^{T}$ by $M^{T}$ and eliminate $X$. This matrix should theoretically be equal to the identity. However that is impossible because $F^{T} A$ is not invertible. Given a matrix $F$, we have to find a matrix $A$ such that $F^{T} A \approx I_{n_{P}}$. In the following, we will consider two candidates for $A$. The first candidate come from the observation that if $F$ is an independent and identically distributed (iid) Gaussian matrix, we have $\frac{1}{m} F^{T} F \approx I_{n_{P}}$ as showed in [5]. We can then take $A=\frac{1}{m} F$ and we will refer to this type of matrix as type 1 (T1). One other candidate for $A$ is obtained by solving the following minimization

$$
\underset{A}{\arg \min }\left\|F^{T} A-I\right\|_{F},
$$

where $\|\cdot\|_{F}$ is the Frobenius norm which is basically the Euclidean norm of the vectorized matrix. This is a well know problem involving the pseudo-inverse of $A$. One can show that the solution writes $A=\left(F F^{T}\right)^{-1} F^{T}$. However, that minimization does not help us determining $F$. In fact, we can show that if $F$ is of full rank, the norm $\left\|F^{T}\left(F F^{T}\right)^{-1} F-I_{p}\right\|_{F}$ is constant and is equal to $\sqrt{n_{P}-m}$. Among all matrix of full rank $F$, some are obviously better than others for a sensing matrix. For example, the matrix

$$
\left(\begin{array}{ll}
I_{m} & 0
\end{array}\right),
$$

is a very bad one because the distance to $I_{p}$ is concentrated in a few entries. We would like to have the error shared equally by all the entries of $F$. Keeping $\left(F F^{T}\right)^{-1} F$ as a possible candidate, we are now interested in the minimization

$$
\underset{\lambda}{\arg \min }\left\|\lambda F^{T}\left(F F^{T}\right)^{-1} F-I\right\|_{\infty} .
$$

The solution is the right scaling of the candidate $\left(F F^{T}\right)^{-1} F$ so as to minimize the maximum error. If $F$ is iid Gaussian, we

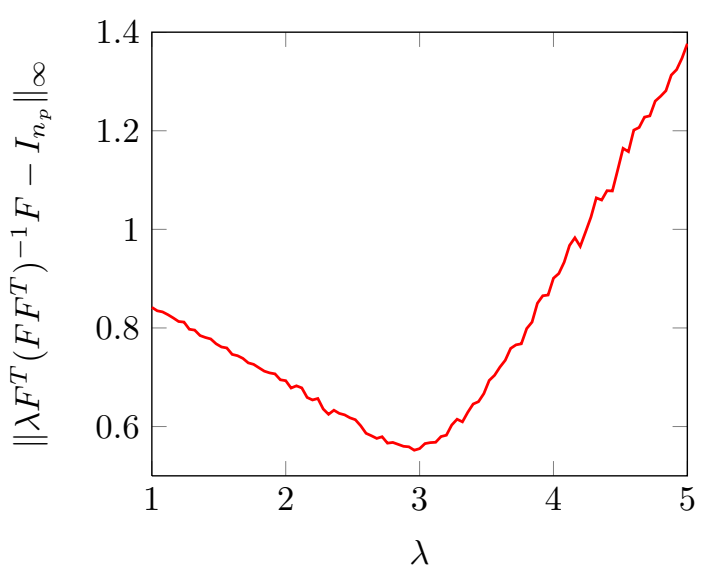

Fig. 1: $\lambda$ minimizing maximum error

have already seen that $F F^{T} \approx n_{P} I_{m}$. We have also $F^{T} F \approx$ $m I_{n_{P}}$. This suggests that $\lambda=\frac{n_{P}}{m}$. This is indeed what we find in Fig. 1 where $m=30$ and $n_{P}=100$, the minimum error is at $\lambda \approx 3=\frac{n_{P}}{m}$.

We have two candidates. Type 1 (T1) is $\frac{1}{m} F$ and type 2 (T2) is $\frac{n_{P}}{m}\left(F F^{T}\right)^{-1} F$. Figure 2 depicts the maximum error for the two types of matrix $A$. We also try two types of sensing matrix $F$ : iid Gaussian and iid Gaussian circulant. Circulant matrix are used as sensing matrix because it has been shown to be almost as effective as the Gaussian random matrix for CS encoding/decoding [6], [7]. Even if candidate of type 1 come from a minimization of the Frobenius norm, it is actually performing better than type 2. Quite surprisingly, the smallest error is obtained when $F$ is the first $m$ rows of a circulant matrix generated from a iid Gaussian vector.

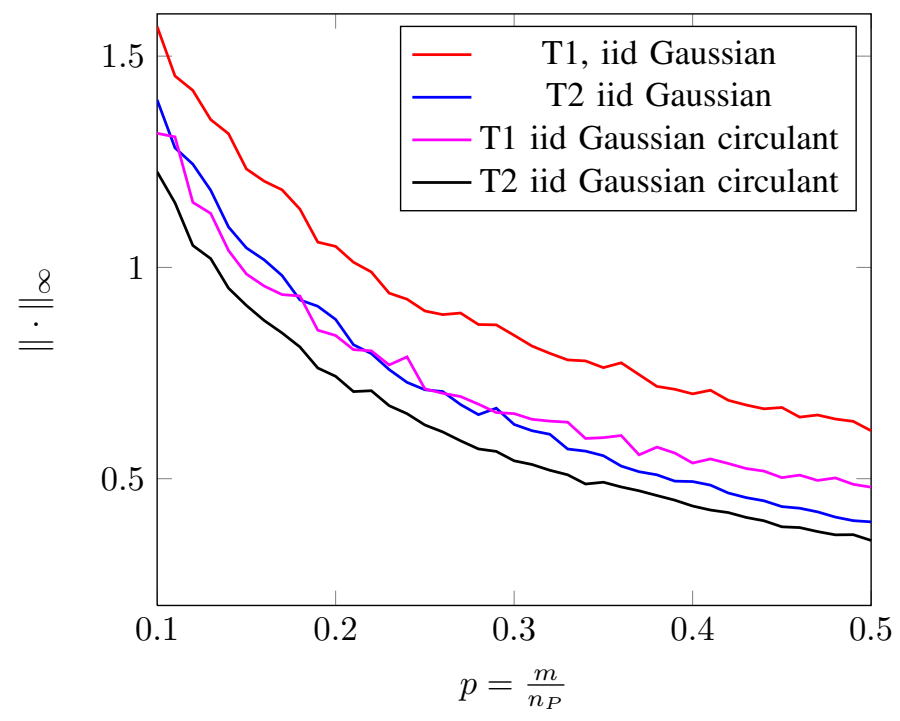

Fig. 2: Maximum error selected candidate for $A$

From now on, we will consider matrix $A$ of type 2 . We can now eliminate $X$ from $X^{T} u=s$ and $M=F X$. We have

$$
X^{T}\left(\frac{n_{P}}{m} F^{T}\left(F^{T} F\right)^{-1}\right) F u=s,
$$


and then, using $M=F X$, we have

$$
M^{T}\left(F F^{T}\right)^{-1} F u=\frac{m}{n_{P}} s .
$$

The minimization then becomes

$$
\underset{u \geq 0}{\arg \min }\|u\|_{1} \quad \text { s.t. } \quad\left\|M^{T}\left(F F^{T}\right)^{-1} F u-\frac{m}{n_{P}} s\right\|_{2}<\sigma .
$$

\section{NUMERICAL RESULTS}

In this section we illustrate compressive template matching by solving minimization (5). We solve that minimization for different regularizers to improve the results. For example, we mix the total variation norm $(T V)$ [8] that adds a geometric constraint that well characterize the location of the signature and the $L_{1}$ norm that promotes sparsity.

Due to their simplicity and flexibility, we use split Bregman algorithms [9], [10] implemented in Matlab ${ }^{\circledR}$ to solve these minimizations. The detailed algorithm is shown below. The non-negativity of $u$ is obtained by forcing intermediate solutions to be non-negative. The Bregman parameters $\beta_{1}$ and $\beta_{2}$ control the two inner Bregman iterations. The size of the

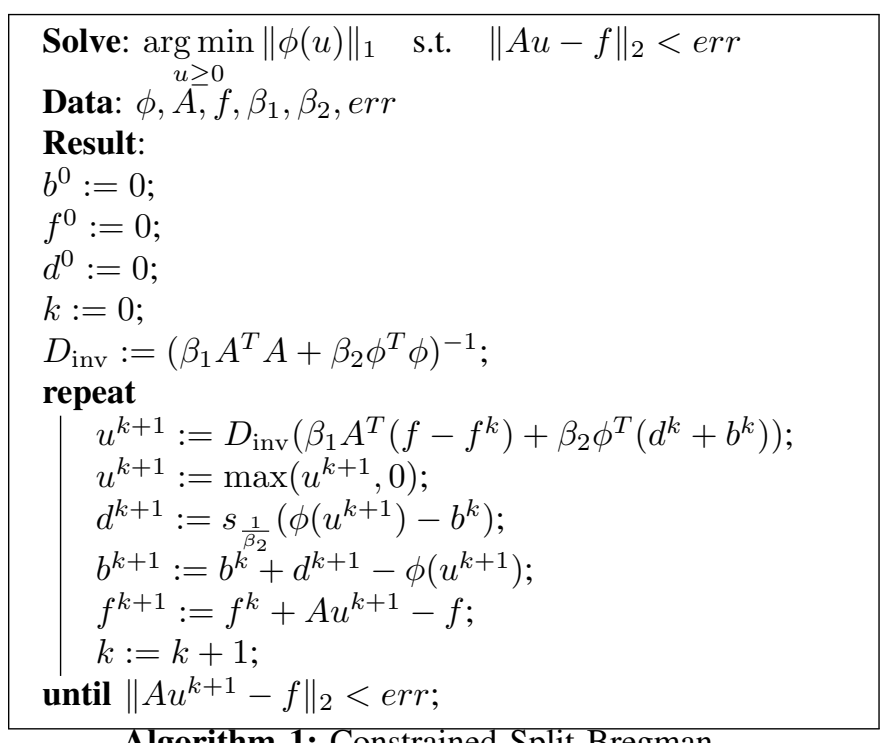

Algorithm 1: Constrained Split Bregman

image is limited to $64 \times 64$ because we choose an iid Gaussian sensing matrix and we have to compute $M^{T}\left(F F^{T}\right)^{-1} F^{T}$. A solution is to store the matrix $\left(F F^{T}\right)^{-1} F^{T}$ once it is computed and reuse it. Another workaround is to use a particular structure on the matrix $F$ so that $\left(F F^{T}\right)^{-1}$ is easy to compute. For example, we can orthogonalize $F$ before we use it.

Another reason is that we have to first compute the matrix $D_{\text {inv }}$. This computational problem can be overcome by using iterative algorithms such as Gauss-Seidel [9] which avoids inverting a huge matrix.

Algorithms are tested on two multispectral images. The first is a $64 \times 64$ multispectral image of 16 bands extracted from a multispectral image database ${ }^{1}$ of everyday objects presented

\footnotetext{
${ }^{1}$ Available at http://www2.cmp.uea.ac.uk/Research/ compvis/MultispectraldB.htm.
}

in [11]. The spectral signature $s$ we want to detect is extracted from Sylvester's nose.

The second multispectral image is extracted from the Moffet Field AVIRIS multispectral image ${ }^{2}$. We selected 16 bands from the 224 available and extracted a $64 \times 64$ image of interest.

\section{A. Test results on the Sylvester multispectral image}

Figure 3 a shows the original image in false color. The result of the template matching algorithm (1) is shown in Fig. 3b. Here, using a $L_{1}$ regularizer is sufficient to have a good detection. In the second row of Fig. 3 we see how compressive

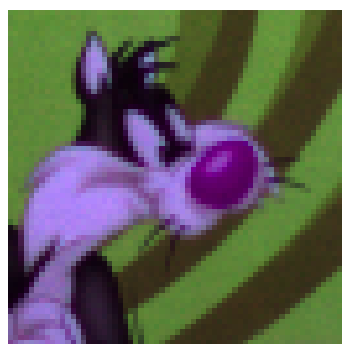

(a) Sylvester image in false color

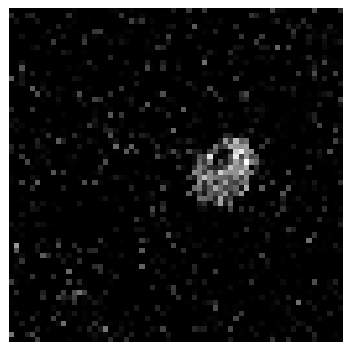

(c) Compressive template matching, $30 \%$ of data, L1 regularizer

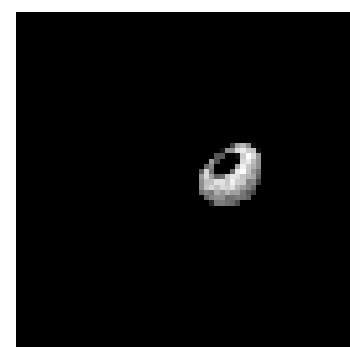

(b) Template matching on Fig. a

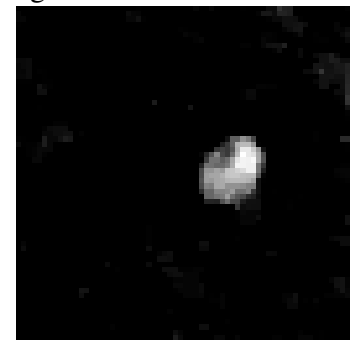

(d) Compressive template matching, $30 \%$ of data, TV/L1 regularizer
Fig. 3: Signature detection on Sylvester image

template matching is performing. The measurement rate is set to $30 \%$. In Fig. 3c, the algorithm (5) has a poor detection. The $L_{1}$ regularizer is not sufficient. If we add a geometric regularizer such as the $T V$ norm, the results are improved as we can see in Fig. 3d.

In Fig. 4, we see how the different algorithms are doing when the image is contaminated with Gaussian noise $(\sigma=$ $15 \%)$.

\section{B. Test results on AVIRIS image}

We then test our algorithm on a AVIRIS image in Fig. 5a. We would like to detect the spectral signature of buildings which is known. We still take $30 \%$ of the overall data and we test the same regularizers as in the previous figures. The template matching in Fig. 5b is still doing well. The compressive template matching with a $L 1$ regularizer in Fig. $5 \mathrm{c}$ is clearly insufficient here. The results are improved if we add a $T V$ regularizer but they are not comparable to the results

\footnotetext{
${ }^{2}$ Available at http://aviris.jpl.nasa.gov/html/aviris. freedata.html
} 

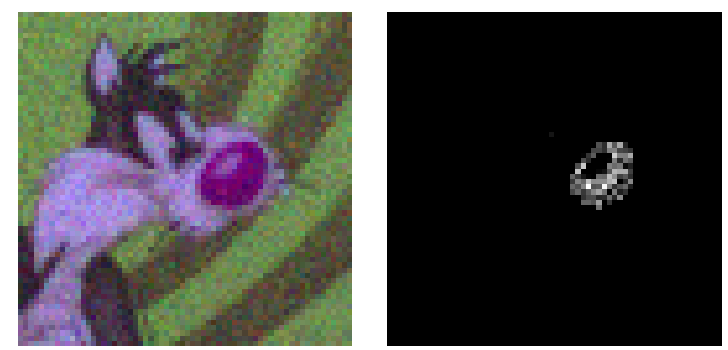

(a) [Sylvester image in false

(b) Template matching on color Fig. a
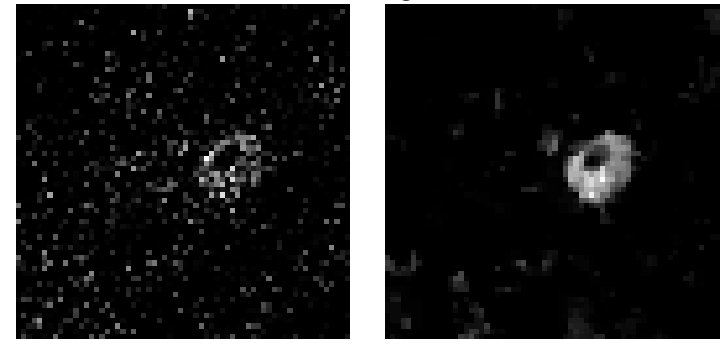

(c) Compressive template (d) Compressive template matching, $30 \%$ of data, L1 matching, $30 \%$ of data, regularizer

TV/L1 regularizer

Fig. 4: Signature detection on Sylvester image

obtained by the template matching algorithm in Fig. 5b. The table I shows the percentage of wrong detection for different measurement rates. A $100 \%$ measurement rate simply means that the standard non-compressive algorithm is used.

\begin{tabular}{|c|c|}
\hline Measurement rate (\%) & Wrong detection (\%) \\
\hline \hline 5 & 5.46 \\
\hline 7 & 4.87 \\
\hline 10 & 3.89 \\
\hline 15 & 3.50 \\
\hline 20 & 3.11 \\
\hline 25 & 2.88 \\
\hline 30 & 2.80 \\
\hline 35 & 2.83 \\
\hline 40 & 2.71 \\
\hline 100 & 0.34 \\
\hline
\end{tabular}

TABLE I: Percentage of wrong detection for various measurement rate

\section{CONCLUSION}

In this paper we propose a compressed sensing scheme for signature detection in multispectral images. Based on the recent $L_{1}$-based algorithm found in paper [1], we add a compressed sensing part by working on measurements rather than on the image itself. We have to carefully choose the sensing matrix so that the approximation made is the smallest possible. We use a slightly modified version of the split Bregman to find non-negative solutions to our problem. Due to the high flexibility of the split Bregman algorithms it is easy to add other type of regularizers to improve the results. Future work includes generalization to pattern matching. It is in fact possible to detect the concatenation of all signatures of the pattern.

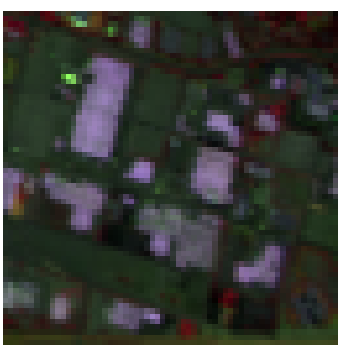

(a) Moffet Field image in false color
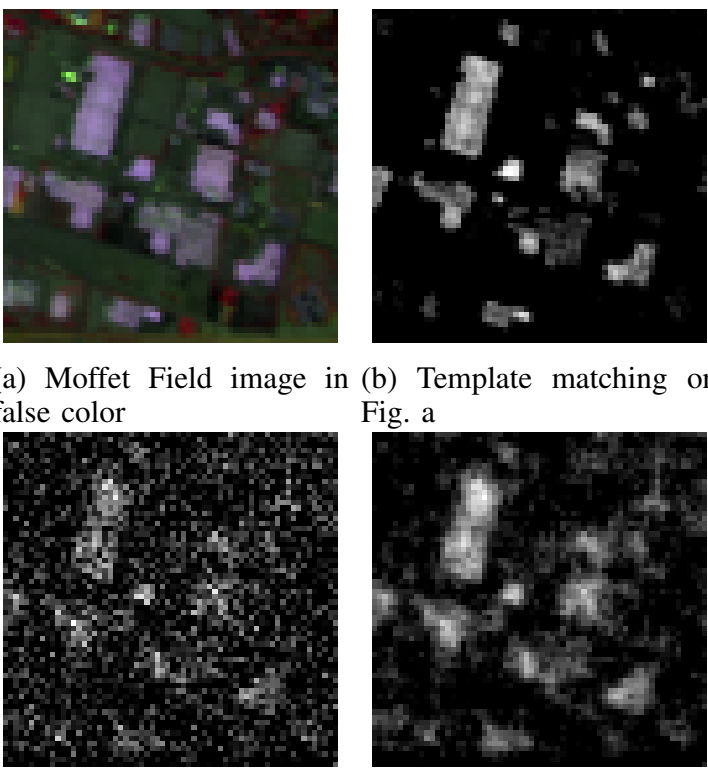

(b) Template matching on Fig. a

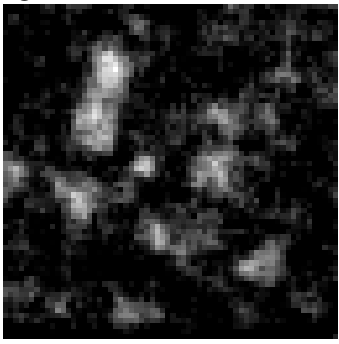

(c) Compressive template (d) Compressive template matching, $30 \%$ of data, L1 matching, $30 \%$ of data, regularizer

TV/L1 regularizer

Fig. 5: Detection of buildings on AVIRIS image

\section{ACKNOWLEDGEMENT}

This work was supported by the french General Directorate for Armament (DGA).

\section{REFERENCES}

[1] Z. Guo and S. Osher, "Template Matching via L1 Minimization and Its Application to Hyperspectral Data," Inverse Problems and Imaging, vol. 5, no. 1, pp. 19-35, 2011

[2] E. Candès, J. Romberg, and T. Tao, "Robust uncertainty principles: Exact signal reconstruction from highly incomplete frequency information," Information Theory, IEEE Transactions on, vol. 52, no. 2, pp. 489-509, 2006.

[3] D. Donoho, "Compressed sensing," Information Theory, IEEE Transactions on, vol. 52, no. 4, pp. 1289-1306, 2006.

[4] C. Li, T. Sun, K. Kelly, and Y. Zhang, "A compressive sensing and unmixing scheme for hyperspectral data processing," Image Processing, IEEE Transactions on, no. 99, pp. 1-1, 2011.

[5] M. Rudelson, "Random vectors in the isotropic position," Journal of Functional Analysis, vol. 164, no. 1, pp. 60-72, 1999.

[6] W. Yin, S. Morgan, J. Yang, and Y. Zhang, "Practical compressive sensing with toeplitz and circulant matrices," Rice University CAAM Technical Report TR10-01, vol. 1, 2010.

[7] J. Romberg, "Compressive sensing by random convolution," SIAM Journal on Imaging Sciences, vol. 2, no. 4, pp. 1098-1128, 2009.

[8] L. Rudin, S. Osher, and E. Fatemi, "Nonlinear total variation based noise removal algorithms," Physica D: Nonlinear Phenomena, vol. 60, no. 1-4, pp. 259-268, 1992.

[9] T. Goldstein and S. Osher, "The Split Bregman Method for L1Regularized Problems," SIAM Journal on Imaging Sciences, vol. 2, p. 323, 2009.

[10] J. Cai, S. Osher, and Z. Shen, "Split bregman methods and frame based image restoration," Multiscale Model. Simul, vol. 8, no. 2, pp. 337-369, 2009.

[11] S. Hordley, G. Finalyson, and P. Morovic, "A multi-spectral image database and its application to image rendering across illumination," in Third International Conference on Image and Graphics. IEEE, 2004 pp. 394-397. 